\title{
ON THE ORDER OF MAGNITUDE OF THE COEFFICIENTS IN TRIGONOMETRIC INTERPOLATION*
}

BY

\section{DUNHAM JACKSON}

1. Introduction. It is well known that there is a remarkable and farreaching parallelism between the properties of the trigonometric interpolating formula determined by the values of a given function at a set of equally spaced points, and those of the Fourier's series for the same function. $\dagger$ This parallelism is closer than the correspondence of sums and integrals in the coefficients would indicate at first sight. Let $f(x)$ be the given function, assumed to have the period $2 \pi$. Let the Fourier's series, to terms of the $p$ th order, be denoted by

$$
s_{p}(x)=\frac{a_{0}}{2}+\sum_{n=1}^{p}\left(a_{n} \cos n x+b_{n} \sin n x\right) .
$$

Its coefficients are

$$
a_{n}=\frac{1}{\pi} \int_{0}^{2 \pi} f(x) \cos n x d x, \quad b_{n}=\frac{1}{\pi} \int_{0}^{2 \pi} f(x) \sin n x d x .
$$

Let the finite trigonometric sum of order $p$ which agrees in value with $f(x)$ at $2 p+1$ points equally spaced over the interval from 0 to $2 \pi$ be

$$
\sigma_{p}(x)=\frac{a_{0 p}}{2}+\sum_{n=1}^{p}\left(a_{n p} \cos n x+b_{n p} \sin n x\right) ;
$$

in particular, it will be assumed that the points of coincidence are the points $x_{k}=2 k \pi /(2 p+1), k=0,1, \cdots, 2 p$. The coefficients here are the sums (1) $\quad a_{n p}=\frac{2}{2 p+1} \sum_{k=0}^{2 p} f\left(x_{k}\right) \cos n x_{k}, \quad b_{n p}=\frac{2}{2 p+1} \sum_{k=0}^{2 p} f\left(x_{k}\right) \sin n x_{k}$.

The resemblance of these to the integrals for $a_{n}$ and $b_{n}$ is apparent; and it is

* Presented to the Society, December 30, 1919.

† Cf., e.g., Bôcher, Introduction to the theory of Fourier's series, An n als of $\mathrm{M}$ a the matics, vol. 7 (1905-06), pp. 81-152; pp. 132-137; Faber, Ueber stetige Funktionen, Mathe mat is che Annale n, vol. 69 (1910), pp. 372-443; pp. 417-443; D. Jackson, On the accuracy of trigonometric interpolation, these Transactions, vol. 14 (1913), pp. 453-461. 
seen that if $n$ is held fast and $p$ increases without limit,

$$
\lim _{n=\infty} a_{n p}=a_{n}, \quad \lim _{n=\infty} b_{n p}=b_{n} .
$$

For any fixed value of $p$, however, if $n$ is nearly equal to $p$, the variations of $\cos n x$ and $\sin n x$ are only very imperfectly represented by the values of these functions at a set of $2 p+1$ points, so that the similarity between $s_{p}$ and $\sigma_{p}$ has to be regarded as a consequence of the special properties of the trigonometric functions, not merely of the definition of an integral as the limit of a sum.

The present paper is concerned with the order of magnitude of the coefficients in the interpolating formula, in case the function $f(x)$ is of limited variation, or has a derivative of limited variation. The method of partial summation is used to give results analogous to those which Picard* obtains for the Fourier's series by the second law of the mean and integration by parts. Incidentally, in the case of a derivative of limited variation, a relatively simple proof is found for the convergence of the Fourier's series, as well as of the interpolating function, to the value $f(x)$.

2. Order of magnitude of the coefficients for a function of limited variation. Let $f(x)$ be a function of period $2 \pi$, having limited variation in any finite interval, its total variation in the interval from 0 to $2 \pi$ being $V$. Then, $\ddagger$ if $a_{n}$ and $b_{n}$ are the coefficients in the Fourier's series for $f(x)$, as above,

$$
\left|a_{n}\right| \leqq \frac{K_{1}}{n}, \quad\left|b_{n}\right| \leqq \frac{K_{1}}{n}, \quad n>0,
$$

where $K_{1}$ is independent of $n$, and may be taken equal to $V / \pi$. This can be seen as follows. The function $f(x)-f(0)$ can be expressed in the form

$$
f(x)-f(0)=f_{1}(x)-f_{2}(x),
$$

where $f_{1}(x)$ and $f_{2}(x)$, the positive and negative variations of $f(x)$ from 0 to $x$, are non-decreasing functions which vanish for $x=0$. Since

$$
f(2 \pi)=f(0) \quad \text { and } \quad f_{1}(2 \pi)+f_{2}(2 \pi)=V,
$$

it follows that

The value of $a_{n}$ is

$$
f_{1}(2 \pi)=f_{2}(2 \pi)=\frac{V}{2} \text {. }
$$

$$
a_{n}=\frac{1}{\pi}\left[\int_{0}^{2 \pi} f_{1}(x) \cos n x d x-\int_{0}^{2 \pi} f_{2}(x) \cos n x d x+f(0) \int_{0}^{2 \pi} \cos n x d x\right] .
$$

* Traité d'analyse, pp. 252-253, 255-256 (second edition).

f It is immediately apparent from the order of magnitude of the coefficients that the Fourier's series is uniformly convergent, but the proof that the sum of the series is identical with $f(x)$ usually requires more attention.

† Cf. Picard, loc. cit., pp. 252-253. 
The third integral in the brackets is zero, for $n>0$. By the second law of the mean,

$$
\int_{0}^{2 \pi} f_{1}(x) \cos n x d x=f_{1}(2 \pi) \int_{\xi}^{2 \pi} \cos n x d x=\frac{V}{2}\left[\frac{\sin n x}{n}\right]_{\xi}^{2 \pi}=-\frac{V}{2} \cdot \frac{\sin n \xi}{n},
$$

where $0 \leqq \xi \leqq 2 \pi$, and hence

Similarly,

$$
\left|\int_{0}^{2 \pi} f_{1}(x) \cos n x d x\right| \leqq \frac{V}{2 n} .
$$

and therefore

$$
\left|\int_{0}^{2 \pi} f_{2}(x) \cos n x d x\right| \leqq \frac{V}{2 n}
$$

$$
\left|a_{n}\right| \leqq \frac{V}{n \pi}
$$

A corresponding computation for $b_{n}$ would seem at first sight to give only the inequality $\left|b_{n}\right| \leqq 2 V /(n \pi)$, since the value of $\int \sin n x d x$, extended from an unknown lower limit to $2 \pi$, might be as great as 2 . For any particular value of $n$, however, the integral defining $b_{n}$ can equally well be extended over an interval of length $2 \pi$ beginning at the point $\pi /(2 n)$, and so terminating at a point where $\cos n x$ vanishes; and by expressing $f(x)-f(\pi /(2 n))$ as the difference of two non-decreasing functions which vanish for $x=\pi /(2 n)$, it can be shown that

$$
\left|b_{n}\right| \leqq \frac{V}{n \pi}
$$

An analogous result for the coefficients $a_{n p}, b_{n p}$ is as follows:

Theorem I. If $f(x)$ is a function of period $2 \pi$ which is of limited variation in any finite interval, then

$$
\left|a_{n p}\right| \leqq \frac{L_{1}}{n}, \quad\left|b_{n p}\right| \leqq \frac{L_{1}}{n}, \quad n>0,
$$

where $L_{1}$ is independent of $n$ and $p$.

If $V$ is the total variation of $f(x)$ in the interval from 0 to $2 \pi$, the constant $L_{1}$ can be taken equal to $V$.

It will be convenient to consider the sums

$$
\sum f\left(x_{k}\right) \cos n x_{k} \quad \text { and } \quad \sum f\left(x_{k}\right) \sin n x_{k}
$$

of (1) together, as the real part and the coefficient of the pure imaginary part of the expression

$$
\Sigma_{0}=\sum_{k=0}^{2 p} f\left(x_{k}\right) e^{n x_{k} i} .
$$


Let

then

$$
w_{q}=\sum_{k=0}^{q} e^{n x_{k} i}=\sum_{k=0}^{q} e^{k \cdot[2 n \pi i /(2 p+1)]} \quad(q=0,1, \cdots, 2 p)
$$

$$
\begin{gathered}
\Sigma_{0}=f\left(x_{0}\right) \cdot w_{0}+f\left(x_{1}\right)\left(w_{1}-w_{0}\right)+f\left(x_{2}\right)\left(w_{2}-w_{1}\right) \\
+\cdots+f\left(x_{2 p}\right)\left(w_{2 p}-w_{2 p-1}\right) \\
=\left[f\left(x_{0}\right)-f\left(x_{1}\right)\right] w_{0}+\left[f\left(x_{1}\right)-f\left(x_{2}\right)\right] w_{1} \\
+\cdots+\left[f\left(x_{2 p-1}\right)-f\left(x_{2 p}\right)\right] w_{2 p-1}+f\left(x_{2 p}\right) \cdot w_{2 p} .
\end{gathered}
$$

The last term written down vanishes, because* $w_{2 p}=0$. Let $H$ be a number such that

$$
\left|w_{q}\right| \leqq H
$$

for $q=0,1, \cdots, 2 p-1$. Then

$$
\begin{aligned}
\left|\Sigma_{0}\right| \leqq\left[\left|f\left(x_{0}\right)-f\left(x_{1}\right)\right|+\left|f\left(x_{1}\right)-f\left(x_{2}\right)\right|\right. & \\
& \left.+\cdots+\left|f\left(x_{2 p-1}\right)-f\left(x_{2 p}\right)\right|\right] H,
\end{aligned}
$$

or, as the sum in brackets can not exceed $V$,

$$
\left|\Sigma_{0}\right| \leqq V H \text {. }
$$

It remains to form an estimate of the magnitude of $H$.

By applying to $w_{q}$ the formula for the sum of $q+1$ terms of a geometric progression, it is seen that

$$
w_{q}=\frac{1-e^{[2(q+1) n \pi i(2 p+1)]}}{1-e^{[2 n \pi i(2 p+1)]}} .
$$

The second term in the numerator is represented by a point on the unit circle in the complex plane, so that the absolute value of the numerator can not exceed 2 . It is evident geometrically (and of course can readily be proved analytically) that the absolute value of the denominator is $2 \sin [n \pi /(2 p+1)]$. Since $n /(2 p+1)<\frac{1}{2}$, and since $\sin y / y$ decreases steadily as $y$ increases from 0 to $\pi / 2$,

$$
\sin \frac{n \pi}{2 p+1}=\frac{n \pi}{2 p+1} \cdot \frac{\sin [n \pi /(2 p+1)]}{n \pi /(2 p+1)}>\frac{n \pi}{2 p+1} \cdot \frac{\sin (\pi / 2)}{\pi / 2},
$$

that is,

$$
\sin \frac{n \pi}{2 p+1}>\frac{2 n}{2 p+1}
$$

* This is a consequence of well-known properties of the sine and cosine, and, in particular, can be inferred immediately from the formula (4) below. 
It is found consequently that

$$
\left|w_{q}\right| \leqq \frac{2 p+1}{2 n},
$$

and the quantity on the right can be substituted for $H$ in (3).

Finally, since $a_{n p}$ and $b_{n p}$ are respectively the real part and the coefficient of the pure imaginary part of $2 \Sigma_{0} /(2 p+1)$,

$$
\left|a_{n p}\right| \leqq \frac{V}{n}, \quad\left|b_{n p}\right| \leqq \frac{V}{n} .
$$

3. Order of magnitude of the coefficients for a function having a first derivative of limited variation. Let it be assumed now that $f(x)$ has a first derivative which is of limited variation in any finite interval, and let $V$ in this case stand for the total variation of the derivative in the interval from 0 to $2 \pi$. By integration by parts, ${ }^{*}$

$$
\begin{gathered}
\int_{0}^{2 \pi} f(x) \cos n x d x=-\frac{1}{n} \int_{0}^{2 \pi} f^{\prime}(x) \sin n x d x, \\
\int_{0}^{2 \pi} f(x) \sin n x d x=\frac{1}{n} \int_{0}^{2 \pi} f^{\prime}(x) \cos n x d x,
\end{gathered}
$$

the additional terms which would otherwise appear on the right vanishing because of the periodicity of the functions involved. Since $f^{\prime}(x)$ satisfies the hypotheses that were imposed on $f(x)$ at the beginning of the preceding section,

and

$$
\left|\int_{0}^{2 \pi} f^{\prime}(x) \sin n x d x\right| \leqq \frac{V}{n}, \quad\left|\int_{0}^{2 \pi} f^{\prime}(x) \cos n x d x\right| \leqq \frac{V}{n},
$$

$$
\left|a_{n}\right| \leqq \frac{V}{n^{2} \pi}, \quad\left|b_{n}\right| \leqq \frac{V}{n^{2} \pi} .
$$

For the case of interpolation, consider once more the sum $\Sigma_{0}$, and its representation by the expression (2). It has already been remarked that $w_{2 p}=0$; let the last term in (2), instead of being dropped, be replaced by

$$
\left[f\left(x_{2 p}\right)-f\left(x_{2 p+1}\right)\right] w_{2 p},
$$

with the understanding that $x_{2 p+1}=2 \pi$. Then, if the general value of $w_{q}$ from (4) is substituted in (2), it appears that

$$
\Sigma_{0}=\frac{1}{1-e^{(2 n \pi i /(2 p+1)]}}\left[\sum_{q=0}^{2 p}\left[f\left(x_{q}\right)-f\left(x_{q+1}\right)\right]-\sum_{q=0}^{2 p}\left[f\left(x_{q}\right)-f\left(x_{q+1}\right)\right] e^{n x_{q}+1 i}\right] .
$$

The first sum on the right reduces to $f\left(x_{0}\right)-f\left(x_{2 p+1}\right)$, which is zero, because

* Cf. Picard, loc. cit., pp. 255-256. 
of the periodicity of $f(x)$. In the second sum, let the subscript $q$ be replaced by $k$, and let

$$
d_{k}=f\left(x_{k+1}\right)-f\left(x_{k}\right) ;
$$

since $x_{q+1}=x_{q}+2 \pi /(2 p+1)$, the expression for $\Sigma_{0}$ reduces to

$$
\Sigma_{0}=\frac{e^{[2 n \pi i /(2 p+1)]}}{1-e^{[2 n \pi i /(2 p+1)]}} \sum_{k=0}^{2 p} d_{k} e^{n x_{k} i} .
$$

This formula corresponds to the result of the integration by parts in the case of the Fourier's coefficients.

In the factor before the sign of summation on the right, the absolute value of the numerator is 1 . The denominator is the one that was considered in the preceding section; its absolute value is $2 \sin [n \pi /(2 p+1)]$, and is greater than $4 n /(2 p+1)$. Consequently

$$
\left|\Sigma_{0}\right| \leqq \frac{2 p+1}{4 n}\left|\sum_{k=0}^{2 p} d_{k} e^{n x_{k} i}\right|
$$

By the mean value theorem,

$$
d_{k}=\left(x_{k+1}-x_{k}\right) f^{\prime}\left(\xi_{k}\right)=\frac{2 \pi}{2 p+1} f^{\prime}\left(\xi_{k}\right), \quad x_{k}<\xi_{k}<x_{k+1} .
$$

Let a function $\phi(x)$ be defined so as to be equal to $f^{\prime}\left(\xi_{k}\right)$ for $x=x_{k}, k=0$, $1, \cdots, 2 p$, equal to $f^{\prime}\left(\xi_{0}\right)$ for $x=2 \pi$, linear between any two successive points $2 k \pi /(2 p+1)$, and periodic with the period $2 \pi$. Then $\phi(x)$ is of limited variation in any finite interval, its graph being made up of a finite number of linear segments, and so satisfies the conditions imposed on $f(x)$ at the beginning of the preceding section. Its total variation from $x=0$ to $x=2 \pi$ is

$$
\begin{aligned}
& \left|f^{\prime}\left(\xi_{1}\right)-f^{\prime}\left(\xi_{0}\right)\right|+\left|f^{\prime}\left(\xi_{2}\right)-f^{\prime}\left(\xi_{i}\right)\right|+\cdots \\
& \quad+\left|f^{\prime}\left(\xi_{2 p}\right)-f^{\prime}\left(\xi_{2 p-1}\right)\right|+\left|f^{\prime}\left(\xi_{0}+2 \pi\right)-f^{\prime}\left(\xi_{2 p}\right)\right|,
\end{aligned}
$$

and this quantity, while not necessarily equal to $V$, is at any rate not greater than $V$. Hence the reasoning of the earlier section is applicable, and it appears that

$$
\left|\sum_{k=0}^{2 p} \phi\left(x_{k}\right) e^{n x_{k^{k}}}\right| \leqq V \cdot \frac{2 p+1}{2 n} .
$$

From this can be deduced successively the inequalities

$$
\begin{aligned}
& \left|\sum_{k=0}^{2 p} d_{k} e^{n x_{k}}\right| \leqq \frac{V \pi}{n}, \quad\left|\Sigma_{0}\right| \leqq \frac{(2 p+1) V \pi}{4 n^{2}}, \\
& \left|a_{n p}\right| \leqq \frac{V \pi}{2 n^{2}}, \quad\left|b_{n p}\right| \leqq \frac{V \pi}{2 n^{2}} .
\end{aligned}
$$


The conclusion may be stated as follows:*

THEOREM II. If $f(x)$ is a function of period $2 \pi$ which has a first derivative. $f^{\prime}(x)$ of limited variation in any finite interval, then

$$
\left|a_{n p}\right| \leqq \frac{L_{2}}{n^{2}}, \quad\left|b_{n p}\right| \leqq \frac{L_{2}}{n^{2}}, \quad n>0,
$$

where $L_{2}$ is independent of $n$ and $p$.

If $V$ is the total variation of $f^{\prime}(x)$ in the interval from 0 to $2 \pi$, the constant $L_{2}$ can be taken equal to $V \pi / 2$.

4. Proof of convergence. If $f(x)$ satisfies the hypotheses of the preceding section, the inequalities found there for the coefficients $a_{n}, b_{n}$ make it clear that the Fourier's series for $f(x)$ is uniformly convergent. It is not so immediately obvious from first principles that the sum of the series must be $f(x)$. On the other hand, since $a_{n p}$ and $b_{n p}$ depend on $p$ as well as on $n$, it is not evident, even as a result of Theorem II, that the interpolating function $\sigma_{p}(x)$ approaches a definite limit as $p$ becomes infinite; but it is readily seen that if $\sigma_{p}(x)$ does converge uniformly to a limiting function $g(x)$, then $g(x)$ must be identical with $f(x)$. More explicitly, if $\sigma_{p}(x)$ converges to the limit $g(x)$ when $p$ becomes infinite through all integral values, it will converge to the same limit if $p$ is restricted to the values $\left(3^{m}-1\right) / 2$, so that $2 p+1=3^{m}$, $m=1,2,3, \cdots$. But in the latter case, if $x$ is any particular value of the form $2 k \pi /\left(3^{\mu}\right)$, where $\mu$ is any positive integer and $k$ is any integer whatever, $\sigma_{p}(x)$ will be equal to $f(x)$ for $m=\mu$ and for all succeeding values of $m$, because of the properties of $\sigma_{p}(x)$ as an interpolating function, and therefore the value of $g(x)$ at this point must be the same as that of $f(x)$. That is, $g(x)$ is equal to $f(x)$ at all points $x=2 k \pi /\left(3^{\mu}\right)$; and as both $f(x)$ and $g(x)$ are continuous, and these points are everywhere dense, $g(x)$ must be equal to $f(x)$ identically. It will be shown that $\sigma_{p}(x)$ converges uniformly to the same limit as $s_{p}(x)$, and then it will follow, by what has just been said, that both approximating functions converge $\dagger$ to the value $f(x)$.

Let

$$
g(x)=\lim _{p=\infty} s_{p}(x) ;
$$

also, let $\sigma_{n p}(x)$ be the sum of the first terms of $\sigma_{p}(x)$, through those of

* In connection with the value of $L_{2}, \mathrm{cf} . \S 5$ below.

$\dagger$ The truth of this conclusion is well known, for the interpolating function as well as for the Fourier's series, with more general hypotheses as to the function $f(x)$; cf. Faber, loc. cit., and also de la Vallée Poussin, Sur la convergence des formules d'interpolation entre ordonnées équidistantes, Bulletins de l'A cadémie royale de Belgique, Classe des Sciences, 1908, pp. 319-403. The purpose of the present section is to answer the natural question what connection there is between the order of magnitude of the coefficients and the convergence of the interpolating function, and incidentally to call attention to the simplicity of the resulting convergence proof. 
order $n$,

$$
\sigma_{n p}(x)=\frac{a_{0 p}}{2}+\sum_{k=1}^{n}\left(a_{k p} \cos k x+b_{k p} \sin k x\right) .
$$

The convergence of $s_{p}(x)$, as already noted, follows from the inequalities derived in the preceding section for $a_{n}$ and $b_{n}$. In consequence of this convergence, and of the inequalities for $a_{n p}$ and $b_{n p}$, there will exist for every positive $\epsilon$ a subscript $n$ such that

$$
\left|g(x)-s_{n}(x)\right|<\frac{\epsilon}{3}, \quad\left|\sigma_{p}(x)-\sigma_{n p}(x)\right|<\frac{\epsilon}{3},
$$

both inequalities holding for all values of $x$, and the latter one for all values of $p>n$. If this value of $n$ is held fast, and $p$ is allowed to increase without limit, $a_{k p}$ and $b_{k p}$ will approach $a_{k}$ and $b_{k}$ respectively, for all values of $k \leqq n$, $\sigma_{n p}(x)$ will uniformly approach $s_{n}(x)$, and, when $p$ is sufficiently large,

$$
\left|s_{n}(x)-\sigma_{n p}(x)\right|<\frac{\epsilon}{3}
$$

for all values of $x$. By combination of the last three inequalities, it is seen that

$$
\left|g(x)-\sigma_{p}(x)\right|<\epsilon
$$

as soon as $p$ is sufficiently large, and this completes the proof of convergence.

5. Alternative method for a function having a derivative of limited variation. If the convergence of the Fourier's series to the value $f(x)$, in the case that $f(x)$ has a first derivative of limited variation, be assumed as known at the start, inequalities for the order of magnitude of the coefficients in the interpolating formula can be obtained without the use of partial summation. When $f\left(x_{k}\right)$ in (1) is replaced by its expression in terms of the Fourier's series, it is found that*

$$
\begin{aligned}
& a_{n p}=a_{n}+\sum_{\lambda=1}^{\infty}\left(a_{\lambda(2 p+1)+n}+a_{\lambda(2 p+1)-n}\right), \\
& b_{n p}=b_{n}+\sum_{\lambda=1}^{\infty}\left(b_{\lambda(2 p+1)+n}-b_{\lambda(2 p+1)-n}\right) .
\end{aligned}
$$

On the other hand, by the inequalities at the beginning of $\S 3$,

$$
\left|a_{n}\right| \leqq \frac{K_{2}}{n^{2}}, \quad\left|b_{n}\right| \leqq \frac{K_{2}}{n^{2}},
$$

where $K_{2}$ is a constant. Furthermore, since $n \leqq p$,

$$
\lambda(2 p+1)+n>(2 \lambda+1) n, \quad \lambda(2 p+1)-n>(2 \lambda-1) n,
$$

* Cf., e.g., de la Vallée Poussin, loc. cit., p. 383. 
so that

$$
\begin{array}{ll}
\left|a_{\lambda(2 p+1)+n}\right| \leqq \frac{K_{2}}{(2 \lambda+1)^{2} n^{2}}, & \left|b_{\lambda(2 p+1)+n}\right| \leqq \frac{K_{2}}{(2 \lambda+1)^{2} n^{2}}, \\
\left|a_{\lambda(2 p+1)-n}\right| \leqq \frac{K_{2}}{(2 \lambda-1)^{2} n^{2}}, & \left|b_{\lambda(2 p+1)-n}\right| \leqq \frac{K_{2}}{(2 \lambda-1)^{2} n^{2}},
\end{array}
$$

$$
\left|a_{n p}\right| \leqq \frac{2 K_{2}}{n^{2}}\left(1+\frac{1}{3^{2}}+\frac{1}{5^{2}}+\cdots\right)=\frac{L_{2}^{\prime}}{n^{2}}, \quad\left|b_{n p}\right| \leqq \frac{L_{2}^{\prime}}{n^{2}},
$$

$L_{2}^{\prime}$ being independent of $n$.

Since*

$$
1+\frac{1}{3^{2}}+\frac{1}{5^{2}}+\cdots=\frac{\pi^{2}}{8}
$$

and since, by the preceding section, $K_{2}$ may be taken equal to $V / \pi$, the value $V \pi / 4$ is found for $L_{2}^{\prime}$, which is an improvement over the value of $L_{2}$ given in the statement of Theorem II.

If $f(x)$ possesses a derivative of order higher than the first having limited variation, the first derivative is certainly of limited variation, and it follows from $\$ 4$, even if it is not assumed otherwise, that the Fourier's series converges to the value $f(x)$. The method just outlined can be used, with the stronger inequalities for $a_{n}$ and $b_{n}$ which are obtained by integration by parts in this case, to yield corresponding inequalities for $a_{n p}$ and $b_{n p}$.

6. The method of partial summation for a function having a second derivative of limited variation. Although the existence of higher derivatives can be taken into account in the manner indicated at the end of the preceding section, it is not without interest to observe that the method of $\$ \S 2,3$ can be made to apply to this case also. The extension is not entirely trivial, but it will perhaps be sufficient to carry it one stage further. Let $f^{\prime \prime}(x)$, then, be everywhere defined, and of limited variation in any finite interval. It is to be shown that

$$
\left|a_{n p}\right| \leqq \frac{L_{3}}{n^{3}}, \quad\left|b_{n p}\right| \leqq \frac{L_{3}}{n^{3}}
$$

where $L_{3}$ is independent of $n$ and $p$. The more exact determination of the constant is of secondary interest.

Let it be understood now that

$$
x_{k}=\frac{2 k \pi}{2 p+1},
$$

for all integral values of $k$, whether contained in the interval from 0 to $2 p+1$ or not; let

$$
d_{k}^{\prime}=f\left(x_{k+1}\right)-f\left(x_{k}\right)=d_{k},
$$

\footnotetext{
* Cf., e.g., Bromwich, Theory of infinite series, p. 187.
} 
an accent being added to the symbol $d_{k}$ previously used, to indicate that these are first differences, as distinguished from the second differences now to be introduced; and let

$$
d_{k}^{\prime \prime}=d_{k+1}^{\prime}-d_{k}^{\prime} .
$$

The coefficients $a_{n p}$ and $b_{n p}$, apart from the factor $2 /(2 p+1)$, are the components of the complex quantity $\Sigma_{0}$ already considered. If

it follows from (5) that

$$
\Sigma_{1}=\sum_{k=0}^{2 p} d_{k}^{\prime} e^{* x_{k} i}
$$

$$
\Sigma_{0}=\frac{e^{[2 \pi \pi t /(2 p+1)]}}{1-e^{[2 \pi \pi i / 2 p+1)]}} \Sigma_{1} .
$$

The sum $\Sigma_{1}$ can be rearranged by partial summation, after the same manner as $\Sigma_{0}$. It is found that

$$
\begin{aligned}
\Sigma_{1} & =d_{0}^{\prime} w_{0}+d_{1}^{\prime}\left(w_{1}-w_{0}\right)+\cdots+d_{2 p}^{\prime}\left(w_{2 p}-w_{2 p-1}\right) \\
& =\left(d_{0}^{\prime}-d_{1}^{\prime}\right) w_{0}+\left(d_{1}^{\prime}-d_{2}^{\prime}\right) w_{1}+\cdots+\left(d_{2 p-1}^{\prime}-d_{2 p}^{\prime}\right) w_{2 p-1}+d_{2 p}^{\prime} w_{2 p} ;
\end{aligned}
$$

since $w_{2 p}=0$, the last term may be replaced by $\left(d_{2 p}^{\prime}-d_{2 p+1}^{\prime}\right) w_{2 p}$. In consequence of (4), furthermore,

$$
\Sigma_{1}=\frac{1}{1-e^{[2 n \pi i /(2 p+1)]}}\left[\sum_{q=0}^{2 p}\left(d_{q}^{\prime}-d_{q+1}^{\prime}\right)-\sum_{q=0}^{2 p}\left(d_{q}^{\prime}-d_{q+1}^{\prime}\right) e^{n x_{q+1}}\right] .
$$

The first sum on the right reduces to $d_{0}^{\prime}-d_{2 p+1}^{\prime}$, and this is equal to zero, because $d_{2 p+1}^{\prime}$ is the same as $d_{0}^{\prime}$. Hence

and

$$
\Sigma_{1}=\frac{e^{[2 \pi \pi i /(2 p+1)]}}{1-e^{[2 n \pi t / 2 p+1) !}} \sum_{k=0}^{2 p} d_{k}^{\prime \prime} e^{n \pi_{k}},
$$

$$
\Sigma_{0}=\left(\frac{e^{[2 n \pi t / 2 p+1)]}}{1-e^{[2 n \pi i(2 p+1)]}}\right)^{2} \sum_{k=0}^{2 p} d_{k}^{\prime \prime} e^{n x_{k} t} .
$$

It is clear that a corresponding relation can be obtained for differences of any order. By reason of the inequality previously used for the factor in parentheses,

$$
\left|\Sigma_{0}\right| \leqq\left(\frac{2 p+1}{4 n}\right)^{2}\left|\sum_{k=0}^{2 p} d_{k}^{\prime \prime} e^{n x i}\right| \text {. }
$$

It remains to discuss the order of magnitude of the sum on the right.

By one more partial summation, which it is not necessary to give in detail, it is seen that

$$
\left|\sum_{k=0}^{2 p} d_{k}^{\prime \prime} e^{n x k i}\right| \leqq\left(\left|d_{1}^{\prime \prime}-d_{0}^{\prime \prime}\right|+\left|d_{2}^{\prime \prime}-d_{1}^{\prime \prime}\right|+\cdots+\left|d_{2 p}^{\prime \prime}-d_{2 p-1}^{\prime \prime}\right|\right) H,
$$


where $H$, as in $\S 2$, is an upper bound for $\left|w_{q}\right|$, and may be taken equal to $(2 p+1) /(2 n)$. So, if $X$ stands for the quantity in parentheses,

$$
\left|\Sigma_{0}\right| \leqq \frac{(2 p+1)^{3}}{32 n^{3}} X
$$

and the problem is further reduced to that of treating the expression $X$.

If a function $f(x)$, possessing a second derivative throughout an interval $a \leqq x \leqq c$, vanishes for $x=a$ and for $x=c$, and also at an intermediate point $x=b$, the first derivative $f^{\prime}(x)$ must vanish at some point between $a$ and $b$, and again between $b$ and $c$, and so $f^{\prime \prime}(x)$ must vanish somewhere between $a$ and $c$. If $f(x)$ is not required to vanish at the points $a, b$, and $c$, but $A+B x+C x^{2}$ is the polynomial of the second degree agreeing in value with $f(x)$ at these three points, the second derivative of

$$
f(x)-\left(A+B x+C x^{2}\right)
$$

must vanish somewhere between $a$ and $c$, or, in other words, there is a point at which $f^{\prime \prime}(x)=2 C$. Now let it be assumed further that $b$ is midway between $a$ and $c$, so that $b=a+h, c=a+2 h$, say. Then the second difference of $f(x)$ for the three points,

$$
f(x+2 h)-2 f(x+h)+f(x),
$$

is the same as the corresponding second difference formed for the function $A+B x+C x^{2}$, and the latter second difference is equal to $2 C h^{2}$. That is, since $2 C=f^{\prime \prime}(\xi)$, where $\xi$ has some value between $a$ and $c$, the second difference of $f(x)$ is equal to* $h^{2} f^{\prime \prime}(\xi)$.

In the problem under discussion, where $h=2 \pi /(2 p+1)$,

$$
d_{k}^{\prime \prime}=\frac{4 \pi^{2}}{(2 p+1)^{2}} f^{\prime \prime}\left(\xi_{k}\right)
$$

where $\xi_{k}$ is some point between $x_{k}$ and $x_{k+2}$. Consequently

$$
\begin{aligned}
X=\frac{4 \pi^{2}}{(2 p+1)^{2}}\left[\left|f^{\prime \prime}\left(\xi_{1}\right)-f^{\prime \prime}\left(\xi_{0}\right)\right|+\left|f^{\prime \prime}\left(\xi_{2}\right)-f^{\prime \prime}\left(\xi_{1}\right)\right|\right. \\
\\
\left.+\cdots+\left|f^{\prime \prime}\left(\xi_{2 p}\right)-f^{\prime \prime}\left(\xi_{2 p-1}\right)\right|\right] .
\end{aligned}
$$

If it could be said that $\xi_{0}<\xi_{1}<\xi_{2}<\cdots$, the quantity in brackets could be brought into immediate connection with the total variation of $f^{\prime \prime}(x)$; but it is not clear that the $\xi$ 's do occur exactly in the order of their subscripts. However, since $x_{0}<\xi_{0}<x_{2}, x_{1}<\xi_{1}<x_{3}$, etc., there is no doubt that the pair of points $\left(\xi_{0}, \xi_{1}\right)$ precedes the pair $\left(\xi_{3}, \xi_{4}\right)$, etc., and as the order of the terms in any one difference $\left|f^{\prime \prime}\left(\xi_{k+1}\right)-f^{\prime \prime}\left(\xi_{k}\right)\right|$ is immaterial, it can be

* Cf. Markoff, Differenzenrechnung (Leipzig, Teubner, 1896), p. 21. 
asserted that for present purposes the points $\xi_{0}, \xi_{1}, \xi_{3}, \xi_{4}, \xi_{6}, \xi_{7}, \cdots$, are essentially in order of algebraic magnitude. Moreover, while there may be a question whether the points $\xi_{0}, \cdots, \xi_{2 p}$ are all included in an interval of length $2 \pi$, they are at any rate all included in an interval of length $4 \pi$. So, if $V$ is the total variation of $f^{\prime \prime}(x)$ in an interval of length $2 \pi$, it is certain that $\left|f^{\prime \prime}\left(\xi_{1}\right)-f^{\prime \prime}\left(\xi_{0}\right)\right|+\left|f^{\prime \prime}\left(\xi_{4}\right)-f^{\prime \prime}\left(\xi_{3}\right)\right|+\left|f^{\prime \prime}\left(\xi_{7}\right)-f^{\prime \prime}\left(\xi_{6}\right)\right|+\cdots \leqq 2 V$. Similarly,

$$
\begin{aligned}
& \left|f^{\prime \prime}\left(\xi_{2}\right)-f^{\prime \prime}\left(\xi_{1}\right)\right|+\left|f^{\prime \prime}\left(\xi_{5}\right)-f^{\prime \prime}\left(\xi_{4}\right)\right|+\cdots \leqq 2 V, \\
& \left|f^{\prime \prime}\left(\xi_{3}\right)-f^{\prime \prime}\left(\xi_{2}\right)\right|+\left|f^{\prime \prime}\left(\xi_{6}\right)-f^{\prime \prime}\left(\xi_{5}\right)\right|+\cdots \leqq 2 V .
\end{aligned}
$$

It would be possible to do something toward replacing these by a stricter set of inequalities, but that point will not be pressed. By combination of the three as they stand,

With (6), this gives

$$
\begin{gathered}
\left|f^{\prime \prime}\left(\xi_{1}\right)-f^{\prime \prime}\left(\xi_{0}\right)\right|+\left|f^{\prime \prime}\left(\xi_{2}\right)-f^{\prime \prime}\left(\xi_{1}\right)\right|+\cdots \leqq 6 V, \\
X \leqq \frac{24 \pi^{2} V}{(2 p+1)^{2}} .
\end{gathered}
$$

$$
\left|\Sigma_{0}\right| \leqq \frac{3 \pi^{2} V}{4} \cdot \frac{2 p+1}{n^{3}} .
$$

Finally, because of the factor $2 /(2 p+1)$ in $a_{n p}$ and $b_{n p}$,

$$
\left|a_{n p}\right| \leqq \frac{3 \pi^{2} V}{2 n^{3}}, \quad\left|b_{n p}\right| \leqq \frac{3 \pi^{2} V}{2 n^{3}},
$$

the factor $3 \pi^{2} V / 2$ being independent of $n$ and $p$.

There is no further essential difficulty in carrying the discussion on to derivatives of higher order.

The University of Minnesota,

Minneapolis, Minn. 